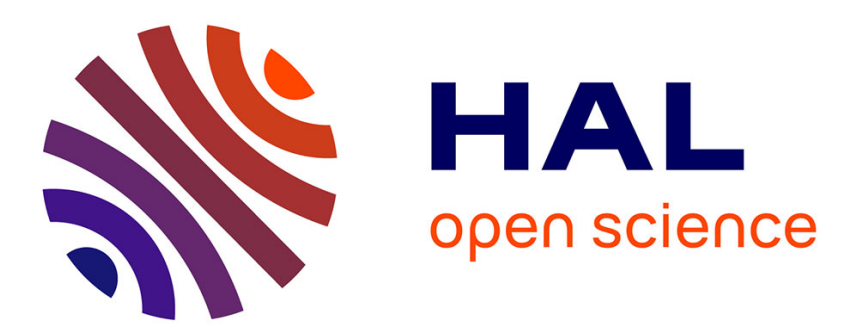

\title{
Impacts des TIC sur la qualité des apprentissages des étudiants et le développement professionnel des enseignants : vers une approche systémique
}

\author{
Marcel Lebrun
}

\section{- To cite this version:}

Marcel Lebrun. Impacts des TIC sur la qualité des apprentissages des étudiants et le développement professionnel des enseignants : vers une approche systémique. STICEF (Sciences et Technologies de l'Information et de la Communication pour l'Éducation et la Formation), 2011, 18, 20 p. hal-00696443

\section{HAL Id: hal-00696443 https://hal.science/hal-00696443}

Submitted on 11 May 2012

HAL is a multi-disciplinary open access archive for the deposit and dissemination of scientific research documents, whether they are published or not. The documents may come from teaching and research institutions in France or abroad, or from public or private research centers.
L'archive ouverte pluridisciplinaire HAL, est destinée au dépôt et à la diffusion de documents scientifiques de niveau recherche, publiés ou non, émanant des établissements d'enseignement et de recherche français ou étrangers, des laboratoires publics ou privés. 
Sciences et Technologies

de I'Information et

\title{
Impacts des TIC sur la qualité des apprentissages des étudiants et le développement professionnel des enseignants : vers une approche systémique.
}

\author{
Marcel LEBRUN (UCL, Louvain-la-Neuve, Belgique)
}

- RESUMÉ : Dans cette synthèse, nous proposons diverses approches méthodologiques, illustrées d'applications concrètes dans le contexte de la mise en place de dispositifs hybrides sur une plateforme d'eLearning, afin de mieux comprendre les rapports systémiques entre des configurations technologiques (outils, usages) et leurs impacts sur l'apprentissage des étudiants et sur le développement professionnel des enseignants du supérieur. Des pistes sont ouvertes afin de mieux comprendre ces rapports interactifs et de définir ainsi des perspectives pour des recherches plus fines et mieux circonstanciées.

- MOTS CLÉS : eLearning, impacts, apprentissage, enseignement, méthodologies

- ABSTRACT : In this paper, we propose different methodological approaches, illustrated by concrete results from observations of hybrid learning environments on a LMS platform, in order to better understand reciprocal influences between technological setups (tools, uses) and their impacts on students learning and on teachers professional development in higher education. Tracks are opened in order to better understand these global interactions and to promote consecutive narrower researches.

- KEYWORDS : e-Learning, impacts, learning, teaching, methodology

○ 1 . Introduction

- 2. Présentation des recherches effectuées

○ 3 . Résultats principaux

○ 4 . Conclusions

○ BIBLIOGRAPHIE et WEBOGRAPHIE

\section{Introduction}

Cela fait une trentaine d'années, depuis les débuts de l'EAO (Enseignement Assisté par Ordinateur) dans les années 80 jusqu'à nos jours, que les rapports entre les technologies et les pédagogies font couler beaucoup d'encre. Les potentiels des technologies éducatives, des logiciels d'apprentissage aux plateformes d'eLearning en passant par le cédérom interactif, ont vite fait croire qu'une couche technologique rajoutée aux formes d'enseignement habituel allait produire l'amalgame salutaire au renouvellement d'une école en quête de résonance avec une société elle-même en quête de savoir-faire, de compétences ... Une école et une société, toutes deux également confrontées au renouvellement et à l'accroissement rapides des savoirs, aux nécessités de l'apprendre toute la vie durant, aux pressions socioéconomiques aussi, etc.

Les recherches les plus fréquentes, souvent construites sur une comparaison « avec et sans technologie » et axées sur les effets en termes de « réussite» des apprenants dans un contexte limité (par exemple : une institution donnée, un outil particulier, une discipline spécifique) ont la plupart du temps été marquées par un no significant difference, un phénomène amplement relevé dans la littérature (Russell, 2009). Remarquons que ces effets en demi-teinte peuvent être étendus à des recherches " avec telle ou telle méthode pédagogique comparée à l'enseignement dit traditionnel ». Les méta-recherches pionnières de Kulik et al. allaient également déjà dans ce sens, les légères différences observées entre les dispositifs étant bien souvent entachées de variance importante ou alors noyées dans un bruit de fond lié à la variété des disciplines, aux différentes méthodes pédagogiques encadrant les outils, ainsi qu'aux modalités 
d'évaluations des apprentissages réalisés (Kulik et al. 1980). Environ vingt ans plus tard, Morgan dans son analyse des effets des LMS (Learning Management System) parle de pédagogies accidentelles pour les qualifier (Morgan, 2003).

Les causes les plus probables de ces résultats peu probants et peu décisifs, en termes de stratégie institutionnelle, par exemple, sont selon nous :

- soit que le véhicule technologique n'implique pas nécessairement une refonte des ressources ou de la pédagogie utilisée (le dispositif pédagogique) ... pas plus que le camion qui amène les victuailles au supermarché n'améliore la nutrition d'une communauté (Clark, 1983),

- soit que les objectifs, les méthodes et les évaluations se modifiant par les usages «bien pensés » des TIC (les Technologies de l'Information et de la Communication), la comparaison avec des approches plus traditionnelles est rendue difficile ou caduque,

- soit encore que les effets recherchés (en termes de compétences ou de savoir-être) restent hors de portée des évaluations certificatives encore largement basées sur des compétences de bas niveau comme la restitution ou l'application (Bloom, 1956).

De manière plus générale encore, les méthodes de formation mises en place « autour des outils » restent fortement marquées par l'amateurisme ou l'improvisation alors que les technopédagogues nous disent, depuis longtemps, l'exigence pédagogique nécessaire à l'élaboration de ces dispositifs en ligne, hybride ou à distance : Tardif, en 1996 déjà, proclamait avec raison qu'une pédagogie rigoureuse est une condition incontournable pour que les TIC tiennent leurs promesses (Tardif, 1996). Selon nous, c'est probablement là que réside le problème : les technologies sont certes porteuses de potentiels pour le développement pédagogique mais, afin d'en retirer les valeurs pédagogiques espérées, elles nécessitent d'être encadrées par des dispositifs pédagogiques basés sur des méthodes plus incitatives et interactives, soutenus par de nouveaux rôles des acteurs, enseignants et étudiants, et finalisés au développement des compétences humaines, sociales et professionnelles de ces acteurs (Figure 1).

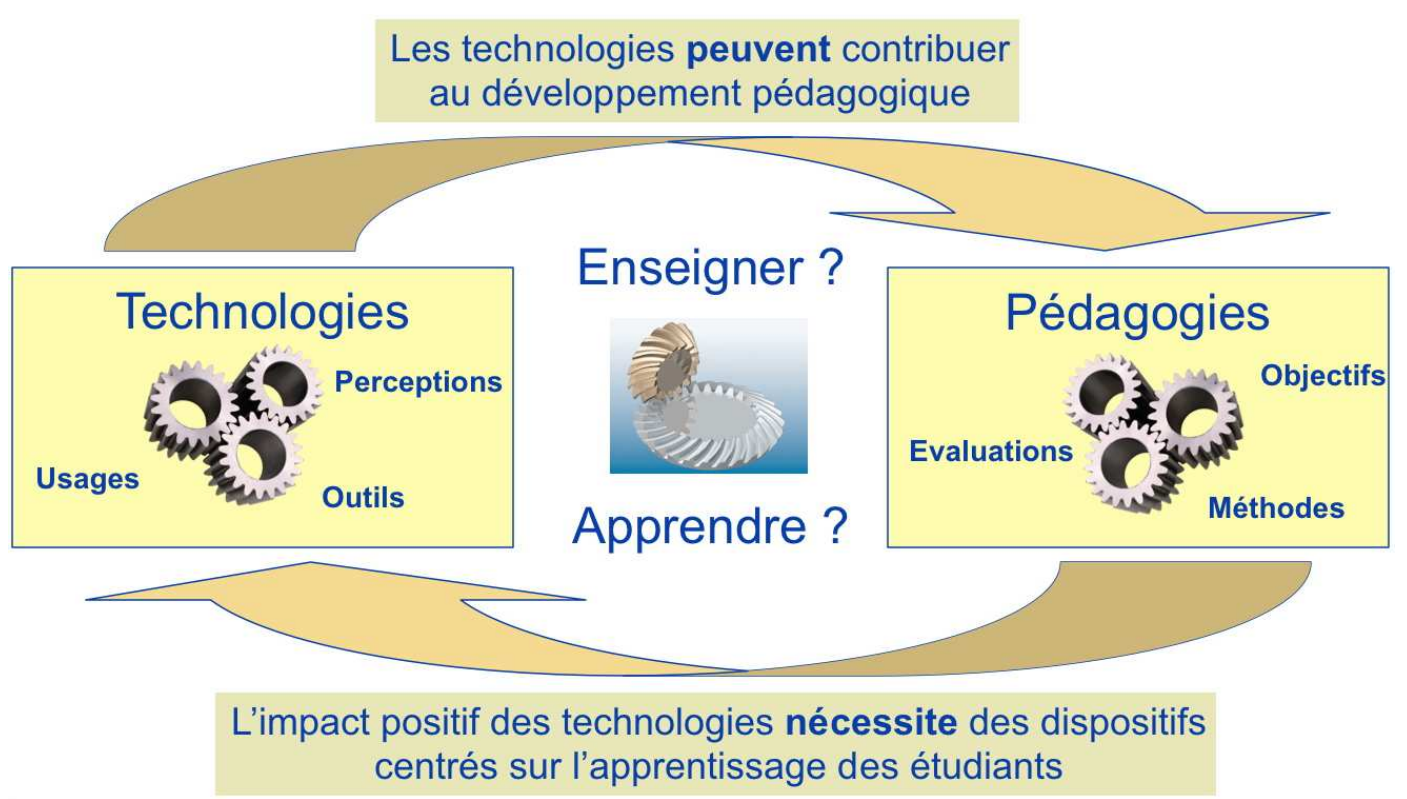

Figure 1. Une représentation systémique des rapports entre technologies et pédagogies

La circularité évoquée dans la figure précédente s'appuie sur des cohérences internes, celle des technologies qui incluent et dépassent l'artefact et l'outil pour devenir instrument de construction des savoirs (Rabardel, 1995), et celle des pédagogies ou plutôt de l'accompagnement pédagogique qui vise à doter les apprenants d'occasions où ils puissent apprendre (Brown et Atkins, 1988). Nous avons illustré les interactions proposées entre les deux pôles de la figure ci-dessus dans un article dont le titre est évocateur : Quality towards an expected harmony: pedagogy and technology speaking together about innovation (Lebrun, 2007). Cet article présente, à différents niveaux (apprentissage, formation, 
institution), cette cohérence pédagogique, globale et systémique, « outils-méthodes-objectifs » à laquelle s'ajoute des modalités d'évaluation des compétences toujours en construction. Elle est proche de l'alignement constructiviste (Biggs, 1996) que nous complétons dans cet article par la prégnance manifeste des technologies dans l'âge de l'information qui caractérise notre troisième millénaire. Il est important de remarquer que ces cohérences souhaitées et ces circularités à mettre en action sont souvent marquées, dans ce document ou ailleurs, de "normativité de bonne intention ou inspirée par des études scientifiques » : les technologies peuvent ..., l'impact positif des technologies nécessite ..., à la condition d'aligner les objectifs, les méthodes ... Cependant, que ce soit sur le pôle " Technologies » ou sur le pôle « Pédagogies », ces intentions, ces objectifs, ces normes sont modulés par les perceptions, les habitudes des acteurs dont nous avons parlé.

Il nous semble finalement intéressant de compléter ce rapide tour d'horizon et de lui donner force en évoquant la démarche Learning Outcomes préconisée par l'Union Européenne en matière d'enseignement supérieur : des objectifs (les compétences que l'étudiant devra développer et dont il devra faire preuve au terme du programme), des méthodes (centrées sur l'activité de l'apprenant mesurée en ECTS, comme, par exemple, l'apprentissage par problèmes, l'apprentissage par projet, l'apprentissage collaboratif ...), des évaluations (formatives et certificatives permettant à l'apprenant de démontrer ce qu'il sait faire) ... Mais, où sont donc, dans ces démarches, les outils, les instruments pourtant évoqués explicitement mais laconiquement sous la compétence de «littéracie numérique » ou implicitement au travers des usages évoqués par des compétences transversales comme chercher et valider l'information, travailler en équipe, savoir communiquer?

Sur le plan de la recherche, ce sont ainsi des méthodes d'investigation de la circularité globale entre technologies et pédagogies que nous préconisons, des approches qui articulent tout à la fois les outils, les usages et les méthodes mises en place " autour de l'outil, les impacts attendus et mesurés sur l'apprentissage, les mobiles (résistances ou incitants) des enseignants et des étudiants utilisateurs de TIC, les impacts sur leur métier, sur leur développement professionnel (Lebrun, 2007). Idéalement, ces recherches s'appuieront sur la collecte d'information auprès des différents acteurs impliqués (responsables institutionnels, gestionnaires TIC, enseignants, étudiants), à différents niveaux d'implication (motivations, outils, usages, instruments cognitifs) et à différents niveaux structurels (institution, gestion des programmes de formation, apprentissages réalisés).

Les plateformes d'eLearning, par la variété des outils proposés et des dispositifs pouvant y être développés, nous paraissent constituer un terrain intéressant pour de telles investigations, un terrain où se rencontrent et se réifient les outils (pôle "Technologies ») et les méthodes de formation (pôle «Pédagogies ») pour devenir des instruments de construction de connaissances et de compétences pour les apprenants, de réflexivité et de développement professionnel pour les enseignants, de promotion et d'innovation dans les institutions. En outre, comme nous assistons à l'heure actuelle, dans la plupart des institutions, à un mélange de dispositifs en présence, hybrides, à distance et ceci tout au long des études des étudiants, nous pouvons espérer mesurer dans ces contextes diversifiés des effets à la fois différentiels et longitudinaux amenés par les TIC.

En ce qui nous concerne, nous entendons par dispositif un ensemble cohérent constitué de ressources (matérielles et humaines), de stratégies, de méthodes et d'acteurs interagissant dans un contexte donné pour atteindre un but. Le but du dispositif pédagogique est de faire apprendre quelque chose à quelqu'un ou mieux (peut-on faire apprendre ?) de permettre à " quelqu'un » d'apprendre " quelque chose » (Lebrun, 2005b). En ce qui concerne l'hybridation, nous la considérons comme un mélange fertile et en proportions variables de différentes modalités de formation, en présence et à distance (Charlier et al. 2006) mais aussi entre des postures d'enseignement transmissif (l'enseignement au sens strict n'exige plus la présence physique en un temps et un lieu donnés, mais peut sortir de l'ex-cathedra pour atteindre l'étudiant où il se trouve) et des postures davantage liées à l'accompagnement de l'apprentissage. Les dispositifs hybrides que nous considérons ici sont ainsi supportés par une plateforme technologique (un rassemblement d'outils) et leur caractère hybride provient d'une modification de leurs constituants (ressources, stratégies, méthodes, acteurs et finalités) par une recombinaison des temps et des lieux d'enseignement et d'apprentissage : il s'agit donc bien d'un continuum dont une dimension est liée au rapport présence-distance et une autre au rapport « enseigner »-« apprendre ». La notion très actuelle de flipped classroom illustre bien cette hybridation. Nous l'avons résumée ainsi : lectures at home and 
homework at school (Lebrun, 2011).

Dans le cadre de ce document, nous souhaitons investiguer de manière empirique l'impact de ces technologies sur l'apprentissage dans différents dispositifs pédagogiques et étayer le fait que les valeurs ajoutées des technologies pour l'apprentissage nécessitent des dispositifs adéquats fondés sur des pédagogies actives, incitatives et interactives. Cette nomenclature nous a été inspirée (Lebrun, 2005b) par les travaux de Lesne qui, outre les modes de travail pédagogique (MTP) à orientation transmissive ou normative, distinguent les MTP de type incitatif à orientation personnelle et de type appropriatif davantage construit sur les interactions sociales (Lesne, 1977).

Notre approche nous paraît essentielle pour compléter une littérature rarissime (au-delà des discours, des enquêtes sur les usages ou la satisfaction des usagers) et éparse sur les effets des TIC sur l'apprentissage en général et sur le développement professionnel (la mutation) des enseignants et pour éviter la part aléatoire de ces impacts que décrivait Morgan : There is little empirical evidence that course management systems actually improve pedagogy. Study findings suggest, however, that using a CMS does invite faculty to rethink their course instruction and instructional environment, resulting in a sort of accidental pedagogy (Morgan, 2003).

Néanmoins, il est opportun de situer les recherches présentées ici comme quelques pièces au sein d'une constellation de travaux précurseurs et qui sont aujourd'hui mutualisés dans le collectif Hy-SUP 1 qui réunit des chercheurs européens de 7 institutions (Burton et al., 2011). Ainsi, parmi d'autres, cette synthèse de nos travaux antérieurs, constitue un modeste jalon temporellement marqué du cheminement vers les recherches actuelles plus amples que nous préconisons.

Après une présentation des recherches effectuées (contexte temporel et institutionnel, cadrage et variables investiguées, modèles de référence et méthodologies de recherche mises en œuvre), les résultats suivants seront analysés : (1) les opinions générales quant aux TIC, entre 2001 et 2006, d'étudiants et d'enseignants, (2) les outils utilisés par ces derniers et leurs usages accompagnés (3) d'une observation longitudinale (entre 2004 et 2007) de ces usages, (4) les effets des TIC, perçus par les uns et les autres, sur l'apprentissage en fonction des dispositifs pédagogiques mis en place. Il s'agira aussi de montrer (5) combien ces effets spécifiques sont dépendants des perceptions générales quant aux TIC et de (6) mettre en évidence, parmi les mobiles déclarés quant aux évolutions pédagogiques perçues, certaines caractéristiques des outils comme leur simplicité ou leur intuitivité.

\section{Présentation des recherches effectuées}

\subsection{Contexte}

C'est dès le début des années 2000, qu'est apparue, à l'Université catholique de Louvain (UCL), l'idée de mettre à la disposition des enseignants un outil qui leur permettrait de déployer facilement des dispositifs pédagogiques à valeur ajoutée pour l'apprentissage. Cette proposition " technique " s'inscrivait dans l'objectif premier de l'IPM (Institut de Pédagogie universitaire et des Multimédias) de favoriser par différentes approches le développement professionnel des enseignants. Après une année (2000-2001) d'utilisation d'une plateforme commerciale choisie pour la multiplicité et la richesse des outils et fonctionnalités qu'elle proposait et gardant en point de mire le souhait d'une appropriation autonome et progressive des enseignants, nous nous sommes rendus à l'évidence suivante : même si la plateforme initialement choisie permettait de réaliser des dispositifs pédagogiques élaborés et complexes, elle rendait les choses simples compliquées, un élément rédhibitoire dans la perspective de promotion et d'évolution de la pédagogie par la technologie. Considérant qu'il n'était pas opportun d'ajouter des difficultés techniques aux déjà difficiles et profondes mutations pédagogiques espérées et nécessaires, il fut alors décidé de mettre à la disposition des enseignants une panoplie d'outils simples (par exemple, dépôt de documents, forum, générateur d'exercices). Le succès fut immédiat en termes d'utilisations, d'usages innovants et de demandes d'accompagnement envers les conseillers technopédagogiques de l'IPM. Claroline (pour Classroom on line) était née sous le nom « local » d'iCampus, l'appellation actuelle de cette plateforme à l'UCL (Lebrun, 2004); (Lebrun, 2005a).

Dans les textes fondateurs (De Praetere, 2003) ; (Lebrun, 2004), nous trouvons l'hypothèse générale 
suivante qui fonde les recherches exposées ici brièvement : une plateforme, simple, intuitive, comme Claroline, devrait permettre aux enseignants de s'occuper de ce qui importe le plus, l'apprentissage des étudiants. Ils devraient ainsi progressivement migrer vers des dispositifs pédagogiques plus riches, plus interactifs, plus proactifs.

Nous souhaitons également mettre en évidence ici un dernier point qui apparaît en filigrane dans ce document. Il s'agit d'une interrogation que nous nous sommes posés très tôt dans le déroulé de l'histoire de l'intégration des TIC dans notre institution: Quelles sont les perceptions " générales ou contextuelles » quant à l'impact des TIC chez nos acteurs, enseignants et étudiants ? S'agit-il du précieux temps que cela prend ? Le coût des équipements ? Le soutien disponible ? En bref, il s'agit de percevoir les moteurs et les freins que nous avons déjà évoqués comme bémol aux circularités et cohérences proposées à la Figure 1. Avant de s'intéresser aux impacts des technologies sur l'apprentissage des étudiants et sur le développement professionnel des enseignants, il est bon de recueillir " l'état d'esprit » de ces acteurs quant à ces dernières.

\subsection{Cadre des recherches présentées}

C'est dès 2001, qu'avec des collègues de l'UCL, nous avons sondé, parmi d'autres éléments contextuels comme les possibilités d'accès et les compétences envers les TIC, les opinions d'étudiants en ce qui concerne l'introduction de TIC dans l'enseignement. Sont-elles considérées comme des freins ou alors comme des moteurs pour l'efficience et l'efficacité de l'enseignement et de l'apprentissage (Reding et al., 2001) ? Des recherches comparables avaient alors également été menées chez les enseignants.

Les mêmes outils méthodologiques utilisés dans ces recherches ont été réutilisés dans les recherches présentées ici (recueil de données en 2006 et 2007) afin de déceler des évolutions éventuelles dans le contexte, l'état d'esprit, l'atmosphère quant aux TIC pourrions-nous dire, des étudiants et enseignants.

Ceci étant dit et considéré comme toile de fond et avec le souhait de structurer davantage les arguments présentés dans l'introduction quant à l'importance du dispositif pédagogique « qui entoure » l'outil, nous $\underline{2}$ nous sommes posés, au cours de ces dernières années, les questions suivantes (qu'il est intéressant de positionner sur la circularité illustrée en Figure 1) :

- quels sont les outils effectivement utilisés par les enseignants dans l'arsenal des outils proposés par la plateforme ? Des outils de transmission, des outils favorisant le travail collaboratif, des outils incitant au travail personnel ? des instruments cognitifs ? (Figure 1 : pôle " Technologies » et potentiel des outils technologiques pour le développement pédagogique)

- observons-nous une évolution dans l'utilisation des outils et dans leurs usages ? En effet, une plateforme simple, intuitive pourrait tout aussi bien conduire à une fossilisation des pratiques qu'à une véritable émancipation des modalités pédagogiques dans notre contexte universitaire ; (Figure 1: pôle « Pédagogies » et nécessité de transformation des pratiques pédagogiques)

- et, but ultime de l'enseignement, la qualité des apprentissages des étudiants est-elle réellement promue, en termes de valeurs ajoutées tout au long de dimensions favorables à ce mécanisme complexe d'appropriation de connaissances et de développement de compétences ? (Figure 1 : interactions constructives entre les deux pôles et valeurs ajoutées sur le plan des apprentissages)

L'hypothèse générale, présentée plus haut, porte ainsi sur plusieurs domaines ou ensembles de variables :

(1) les perceptions générales des étudiants et des enseignants quant aux TIC plongées dans un univers de formation

(2) les outils connus et utilisés ainsi que les usages qui en sont faits par les enseignants et les étudiants,

(3) la possibilité de développer réellement des outils technologiques simples et intuitifs permettant aux enseignants de s'occuper de ce qui importe le plus, l'apprentissage des étudiants,

(4) le développement professionnel des enseignants induit par la mise en place, l'analyse et l'évaluation d'usages progressifs des TIC,

(5) l'impact global sur l'apprentissage des étudiants et finalement, 
(6) la compréhension de cet impact en fonction des outils et des usages proposés par l'enseignant.

Le schéma ci-dessous (Figure 2) articule quelques unes de ces considérations. Nous y avons utilisé le terme « eLearning » pour souligner les articulations recherchées entre le « e » (Pôle « Technologies » de la Figure 1) et « Learning » (Pôle « Pédagogies »).

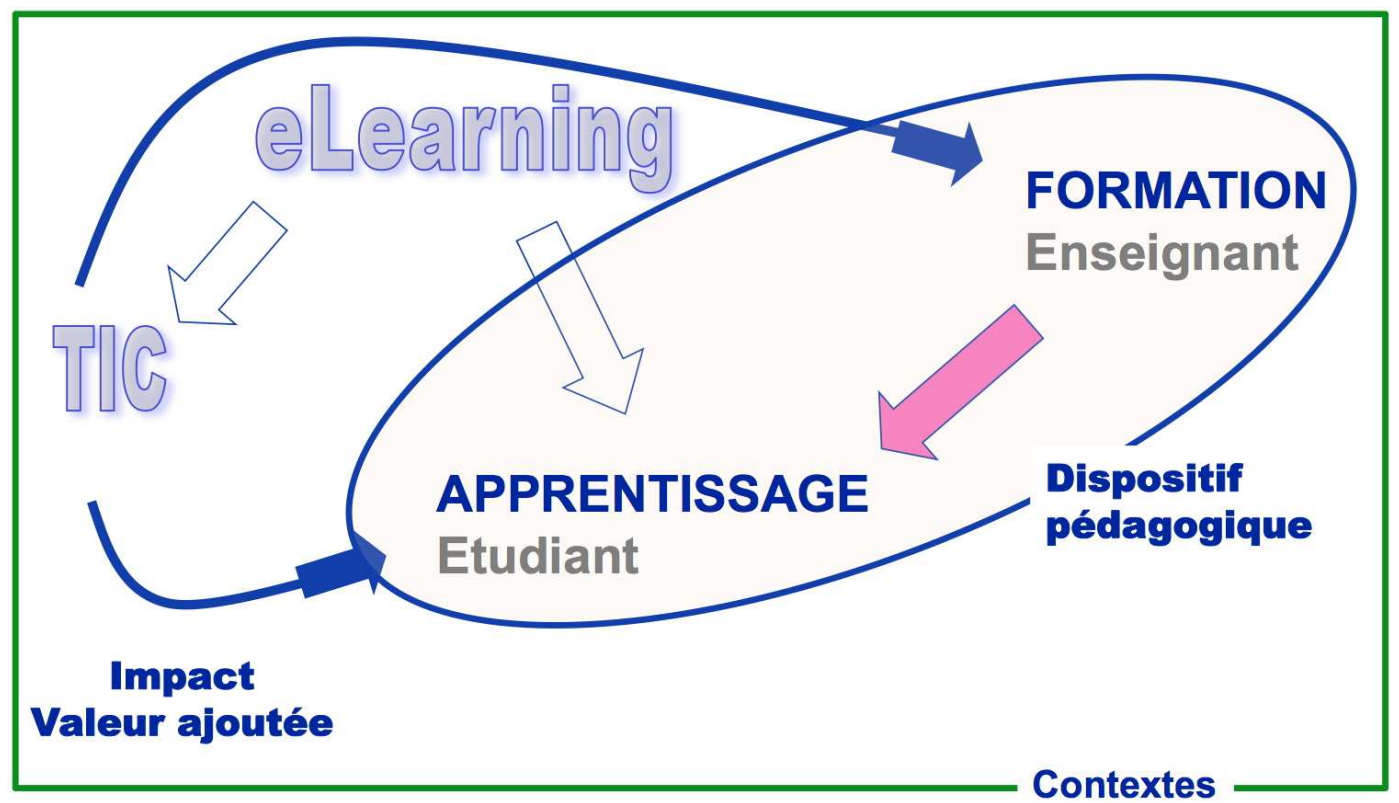

Figure 2. Articulation des variables présentées dans cet article

Dans cet article de synthèse et de perspective, nous ne pouvons présenter dans le détail l'ensemble des recherches que nous menons depuis quelques années dans le contexte de l'enseignement supérieur. Des articles plus ciblés présentent le cadre conceptuel de notre analyse sur les rapports entre technologies et pédagogies (Lebrun, 2007), l'ensemble des données et des analyses effectuées sur les outils, les usages, les perceptions des étudiants quant aux valeurs ajoutées d'apprentissage (Docq et al., 2008), et finalement sur les impacts en termes de développement professionnel des enseignants (Lebrun et al., 2009).

C'est à une mise en perspective, complétée d'analyses rendues nécessaires par cette synthèse et par notre intention méthodologique, que cet article souhaite s'atteler. Il offre un cadre compréhensif (au sens quasi étymologique) à des recherches plus spécifiques, plus localisées ou plus précises portant sur la médiatisation des ressources ou la médiation (Meunier et Peraya, 2004), sur l'apprentissage collaboratif en ligne (Henri et Lundgren-Cayrol, 2001), sur l'hybridation des dispositifs (Charlier et al.. 2006) et la porosité (entre le cadre institutionnel et le cadre de la vie quotidienne) de ces derniers suite à l'émergence du Web 2.0 (Caron et Varga, 2009), sur la formation des enseignants et les postures professionnelles de ces derniers (Lameul, 2008), sur les valeurs ajoutées des TIC (Docq et al., 2010) et les conditions de l'émergence de celles-ci (Barrette, 2005), etc. L'imposant handbook de référence de Voogt et Knezek (Voogt et Knezek, 2008) montre combien il est utile de disposer de cadre élargi afin de mettre en évidence les déterminants, les conditions et les effets des technologies sur l'enseignement et l'apprentissage. Nous tentons d'y contribuer dans cette publication.

\subsection{Modèles de référence}

Mesurer les impacts d'outils technologiques sur l'apprentissage demande de pouvoir jauger leurs effets (usages effectifs, perceptions des apprentissages, etc.) le long de dimensions représentatives de l'apprentissage. Le modèle pragmatique de Lebrun propose différentes dimensions (Lebrun, 2005a) ; (Lebrun, 2007) issues d'une synthèse de travaux qualitatifs portant sur : (1) les compétences telles que postulées par différents acteurs de la Société, (2) les méthodes pédagogiques préconisées par les Sciences de l'éducation afin d'activer les apprentissages et d'ainsi atteindre les objectifs visés et (3) les potentiels des outils technologiques issus des recherches dans le domaine des technologies éducatives. Nous 
retrouvons ici, mais sur un autre plan, l'une des cohérences présentées plus haut, celle entre les objectifs (1), les méthodes (2) et les outils (3). Par exemple, si la compétence au travail d'équipe est abondamment citée par différentes autorités (directions d'institutions, acteurs politiques, Union européenne), l'apprentissage collaboratif peut fournir un terrain d'exercice et de validation de cette compétence et, les outils synchrones et asynchrones ou encore les réseaux sociaux peuvent soutenir cette activité. C'est au travers d'une analyse comparative de ces différents domaines (discours des acteurs, Sciences de l'éducation et recherches sur les TIC) à la recherche de facteurs d'apprentissage communs qu'a été conçu ce modèle pragmatique illustré à la Figure 3.

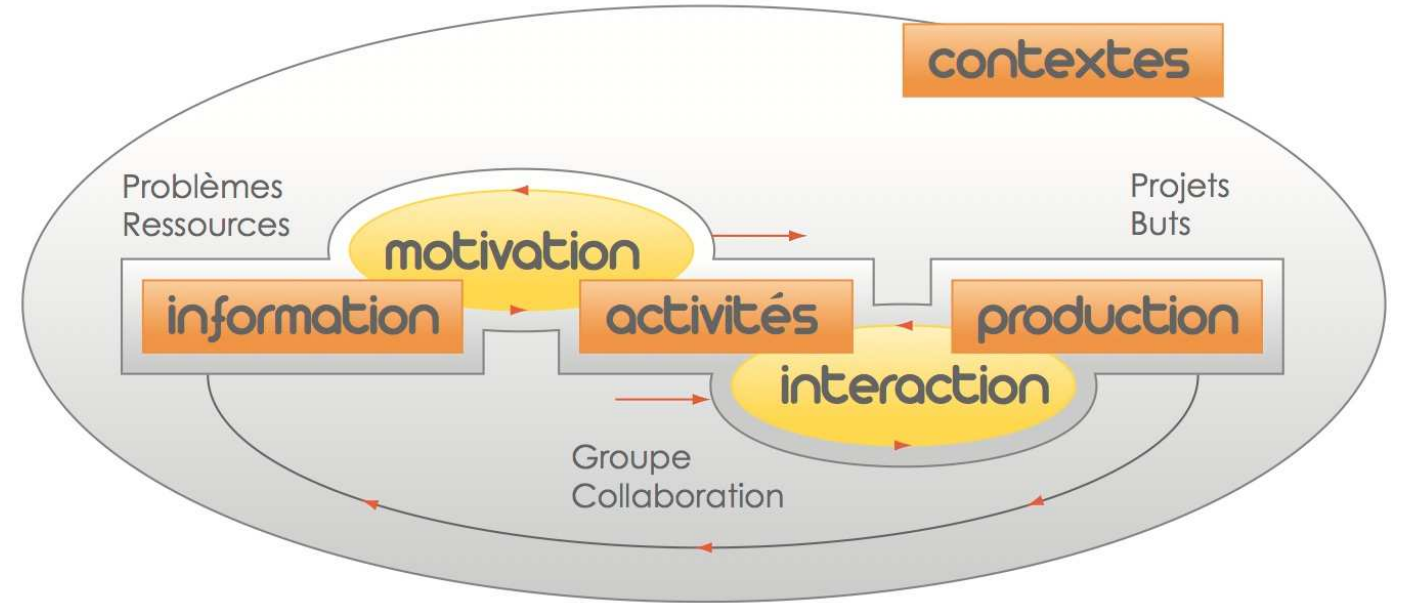

Figure 3. Un modèle pragmatique pour analyser les effets de dispositifs (avec ou sans TIC) sur l'apprentissage

Ce modèle est présenté comme utile pour concevoir et analyser des dispositifs basés sur les technologies et visant des valeurs ajoutées en termes d'apprentissage ainsi que pour évaluer les effets de tels dispositifs. Les cinq dimensions de ce modèle pragmatique sont résumées ici : les activités de l'étudiant (centrales dans le modèle) portent sur des ressources internes (les connaissances déjà-là) et externes (les ressources fournies ou à rechercher) nommées informations dans le modèle et sont orientées vers la production de nouvelles connaissances, de nouvelles compétences. Les moteurs de l'engagement de l'étudiant dans la tâche, de sa persévérance et du soutien formatif à son activité sont nommés dans ce modèle motivation(s) et interaction(s) $\underline{\text { (Lebrun, 2005b) }}$.

En ce qui concerne le développement professionnel des enseignants, il est considéré dans son cheminement de formes transmissives d'enseignement (mode réactif) vers des formes interactives (mode interactif) pour se prolonger vers des formes incitatives (mode proactif) davantage focalisées vers l'attention et le soutien à l'individu apprenant (Lebrun et Viganò, 1995a); (Lebrun et Viganò, 1995b) ; (Lebrun, 2008).

\subsection{Méthodologies de recherche mises en oeuvre}

C'est dès 2000-2001, au début de la réflexion sur l'utilisation de l'Internet dans notre institution et à l'aube de l'émergence des outils qui allaient s'appeler " plateformes d'eLearning ou d'apprentissage en ligne », que nous avons relevé, dans une population de 330 étudiants de l'UCL, les perceptions des freins (les obstacles) et des moteurs (les incitants) quant à l'utilisation des TIC dans la formation. Les outils méthodologiques étaient constitués d'un ensemble de 9 items-obstacles et de 9 items-appuis auxquels était adjointe une échelle d'accord (Reding et al., 2001). Des données (non publiées) relevées, à la même époque et avec les mêmes outils méthodologiques, auprès d'enseignants $(\mathrm{N}=80)$ ont également été récoltées. Nous avons utilisé à nouveau ces mêmes instruments dans les enquêtes de 2006 (chez les étudiants) et de 2007 (chez les enseignants) dont il est question ci-dessous et qui constituent un des objets principaux de cet article.

Cohérents avec l'approche présentée ci-dessus, d'autres instruments de recueil d'informations simultanés avec les précédents de 2006 et 2007 et portant sur les domaines cités plus hauts, ont été menés avec les 
outils méthodologiques suivants :

- l'observation des statistiques d'utilisation des outils auxquelles nous pouvons accéder, d'année en année, au travers des bases de données de la plateforme;

- une enquête effectuée auprès des enseignants utilisateurs de la plateforme iCampus : outre des données signalétiques portant sur le sexe, l'âge, la carrière, la faculté, la discipline ... nous avons recueilli, à propos de l'un de leurs cours, des informations sur les outils utilisés, les usages effectifs et les perceptions quant aux valeurs ajoutées pour l'apprentissage $(\mathrm{N}=153)$;

- une enquête effectuée auprès des étudiants utilisateurs de la plateforme en suivant le même schéma que l'enquête auprès des enseignants et en proposant des items comparables $(\mathrm{N}=1179)$.

Ces enquêtes (réalisées donc à partir de 2006) proposaient principalement des choix simples (les outils utilisés), l'identification des usages principaux au sein d'une liste, des échelles d'accord (pas du tout d'accord, plutôt pas d'accord, plutôt d'accord, tout à fait d'accord) sur des propositions relatives aux valeurs ajoutées perçues pour les apprentissages.

\section{Résultats principaux}

\subsection{Les opinions quant aux TIC en toile de fond.}

Nous présentons ici les résultats (Tableau 1) obtenus en 2001, pour les étudiants ( $\mathrm{N}=330$ ) et les enseignants $(\mathrm{N}=80)$ et en 2006-2007, pour les étudiants (en 2006, $\mathrm{N}=1179)$ et les enseignants (en 2007, $\mathrm{N}=179$ ). Il s'agit en fait du pourcentage d'accord (les réponses plutôt d'accord et tout à fait d'accord rassemblées) avec les 9 items-obstacles (les technologies étant considérées par les répondants comme un obstacle à la formation) et les 9 items-appuis les technologies étant considérées par les répondants comme un appui à la formation).

\begin{tabular}{|l|l|l|l|}
\hline \multirow{2}{*}{} & & $\begin{array}{l}\text { Pourcentage } \\
\text { d'accord } \\
\text { Enseignants - 2007 }\end{array}$ & $\begin{array}{l}\text { Pourcentage } \\
\text { d'accord } \\
\text { Etudiants - 2006 }\end{array}$ \\
\hline \hline \multirow{2}{*}{$\begin{array}{l}\text { TIC }= \\
\text { Obstacles }\end{array}$} & 2001 & $38+/-8 \%$ & $62+/-7 \%$ \\
\cline { 2 - 4 } & $2006-2007$ & $17+/-4 \%$ & $27+/-5 \%$ \\
\hline \hline \multirow{2}{*}{$\begin{array}{l}\text { TIC }= \\
\text { Appuis }\end{array}$} & 2001 & $78+/-5 \%$ & $60+/-9 \%$ \\
\cline { 2 - 4 } & $2006-2007$ & $74+/-2 \%$ & $66+/-9 \%$ \\
\hline
\end{tabular}

Tableau 1. Pourcentages d'accord avec les propositions Obstacles ou Appuis des TIC dans l'enseignement

Dans la limite des barres d'erreur (purement statistique), nous constatons :

- les opinions des enseignants, que ce soit en 2001 ou en 2007, sont généralement plus « enthousiastes » que celles des étudiants : les TIC sont moins considérées comme un obstacle et sont davantage considérées comme un appui.

- une nette diminution par rapport à 2001, chez les enseignants et chez les étudiants, de la perception des TIC considérées comme un obstacle (pratiquement un facteur 2). Chez les enseignants (enquête réalisée en 2007), les deux items-obstacles principaux en 2001 ( le temps que cela prend » $75 \%$ d'accord pour cet obstacle en 2001 et $42 \%$ en 2007 - et « le manque de soutien disponible » - $68 \%$ en 2001 et $29 \%$ en 2007) sont en nette régression. L'item-obstacle qui régresse le plus est celui lié aux compétences nécessaires, allant d'un pourcentage d'accord de $40 \%$ en 2001 à $10 \%$ en 2007 . Chez les étudiants (enquête réalisée en 2006), on remarque une nette diminution des items-obstacles « manque de formation » ( $85 \%$ en 2001 à $27 \%$ en 2006), « aisance face aux outils technologiques » $(60 \%$ en 2001 à 
$14 \%$ en 2006) et une diminution relativement plus faible de l'obstacle lié aux coûts ( $59 \%$ en 2001 à $33 \%$ en 2006).

- une relative stagnation moyenne de la considération des TIC comme un appui à l'enseignement. Chez les enseignants, tous les items-appuis sont en très légère diminution (cependant non significative) sauf un item-appui en progression significative : « pouvoir communiquer avec les étudiants » qui passe de $55 \%$ d'accord en 2001 à 78\% d'accord en 2007. Chez les étudiants, les plus fortes (significatives) augmentations des pourcentages d'accord portent sur les items-appuis suivants : « le gain de temps » (de $46 \%$ en 2001 à $79 \%$ en 2006), " le goût pour la technologie et la création » (de $43 \%$ en 2001 à $65 \%$ en 2006) et « la communication avec l'enseignant» (de 63\% en 2001 à $87 \%$ en 2006).

Plusieurs recherches ont analysé depuis les nôtres ces perceptions des étudiants à propos des TIC (Raby et al., 2011). Nous y ajoutons les points de vue des enseignants, une approche longitudinale et une étude des obstacles à leur utilisation. Nous reviendrons sur ce point plus loin en nous focalisant sur des perceptions plus spécifiques en termes d'enseignement et d'apprentissage. Ainsi, nous verrons au point 3.5 comment ces perceptions globales des effets positifs ou négatifs des TIC, exprimées ici en termes d'opinions, influent ou non sur la perception plus spécifique des effets sur l'apprentissage.

\subsection{Outils et usages}

En ce qui concerne les outils utilisés sur la plateforme par les étudiants et les enseignants, les résultats globaux ne sont guère étonnants. Aux questions (enquêtes de 2006 et 2007), relatives à l'utilisation effective des outils, ce sont les outils de transmission d'informations (par exemple, la description du cours, les documents, les liens, les annonces) qui arrivent en tête avec des fréquences de l'ordre de $80 \%$ des répondants pour les deux groupes. Ensuite, des outils d'interaction (les groupes, les forums) et des outils d'incitation au travail personnel ou de groupe (les exercices, les travaux) se présentent avec des fréquences de 1'ordre de 50\% des répondants (Docq et al., 2008). Des outils plus récents ou alors généralement moins connus (comme le Wiki) ou plus sophistiqués (comme le parcours pédagogique, générateur d'apprentissage plus dirigé) arrivent en queue avec des fréquences de tout au plus $15 \%$.

En ce qui concerne les usages principaux de la plateforme, le tableau ci-dessous (Tableau 2) confirme les résultats tirés des outils. Les résultats en grisé mettent en évidence les trois choix les plus fréquents.

Parmi les usages principaux (on demandait aux répondants de choisir leurs 4 usages principaux), on constate que le trio de tête est le même pour les enseignants et pour les étudiants : la plateforme est surtout utilisée pour diffuser des informations, des notes, des ressources complémentaires.

\begin{tabular}{|l|l|l|}
\hline \multirow{2}{*}{$\begin{array}{l}\text { Tenant compte de tous les cours que } \\
\text { vous avez sur iCampus, vous utilisez } \\
\text { principalement iCampus pour ... }\end{array}$} & $\begin{array}{l}\text { Fréquence de choix de } \\
\text { l'item, en \% }\end{array}$ \\
\cline { 2 - 3 } & Enseignants & Etudiants \\
\hline \hline $\begin{array}{l}\text { 1. Diffuser / consulter des éléments } \\
\text { d'information }\end{array}$ & 77 & 85 \\
\hline $\begin{array}{l}\text { 2. Diffuser / télécharger les notes de } \\
\text { cours }\end{array}$ & 54 & 88 \\
\hline $\begin{array}{l}\text { 3. Communiquer les travaux / envoyer } \\
\text { les travaux au professeur }\end{array}$ & 24 & 23 \\
\hline $\begin{array}{l}\text { 4. Encadrer en ligne les étudiants / } \\
\text { communiquer avec le professeur }\end{array}$ & 19 & 15 \\
\hline $\begin{array}{l}\text { 5. Proposer / réaliser des } \\
\text { auto-évaluations }\end{array}$ & 5 & 6 \\
\hline
\end{tabular}




\begin{tabular}{|l|l|l|}
\hline $\begin{array}{l}\text { 6. Faire travailler les étudiants en } \\
\text { collaboration / travailler en groupe avec } \\
\text { d'autres étudiants }\end{array}$ & 18 \\
\hline $\begin{array}{l}\text { 7. Maintenir la communication en } \\
\text { dehors des activités en classe }\end{array}$ & 41 & 12 \\
\hline $\begin{array}{l}\text { 8. Proposer / consulter des ressources } \\
\text { complémentaires au cours }\end{array}$ & 50 & 44 \\
\hline $\begin{array}{l}\text { 9. Offrir / consulter des ressources qui } \\
\text { ne pourraient être rendues disponibles } \\
\text { autrement (ex. multimédia) }\end{array}$ & 20 \\
\hline $\begin{array}{l}\text { 10. Rendre disponibles / réaliser des } \\
\text { exercices }\end{array}$ & 32 \\
\hline \hline $\begin{array}{l}\text { 11. Rendre disponibles des travaux / } \\
\text { consulter les travaux d'autres étudiants }\end{array}$ & 16 \\
\hline
\end{tabular}

\section{Tableau 2. Usages principaux de la plateforme}

On remarque les plus faibles taux pour les modalités interactives (collaboration et communication) et incitatives (exercices et auto-évaluations) avec des différences notables chez les enseignants (taux d'accord plus élevé) et chez les étudiants (taux plus faible).

Serait-ce une image instantanée ou encore un signe d'une fossilisation ou d'une stagnation des pratiques par les technologies, les enseignants (et les étudiants) ne faisant que reproduire les anciennes pratiques avec les nouveaux outils? Mais qu'en est-il de l'évolution de ces pratiques?

\section{3. Évolution ou stagnation des modalités pédagogiques}

Armés des considérations précédentes relatives à une bonne concordance des distributions des outils et des usages proposés dans le cadre des enquêtes, nous avons consulté les bases de données de la plateforme iCampus quant à l'évolution de l'utilisation des outils au cours des années. Pour cela, nous avons considéré non seulement le fait que l'outil soit activé ou non dans la plateforme mais aussi le degré de son utilisation mesuré par le nombre d'objets présents dans les différents outils : le nombre de ressources dans les dossiers de documents, la quantité d'exercices proposés, le nombre de forums et de sujets dans les forums, le nombre de sessions de travaux ouvertes ... Cela donne un indice global qui permet de se faire une bonne idée des outils mais aussi partiellement et quantitativement des usages. Des détails de cette méthodologie sont explicités par ailleurs (Lebrun et al., 2007).

Dans le graphique ci-dessous (Figure 4), il faut savoir que, entre les années illustrées (2004 et 2007), le nombre de dispositifs de formation ou de cours sur la plateforme en question a doublé. 


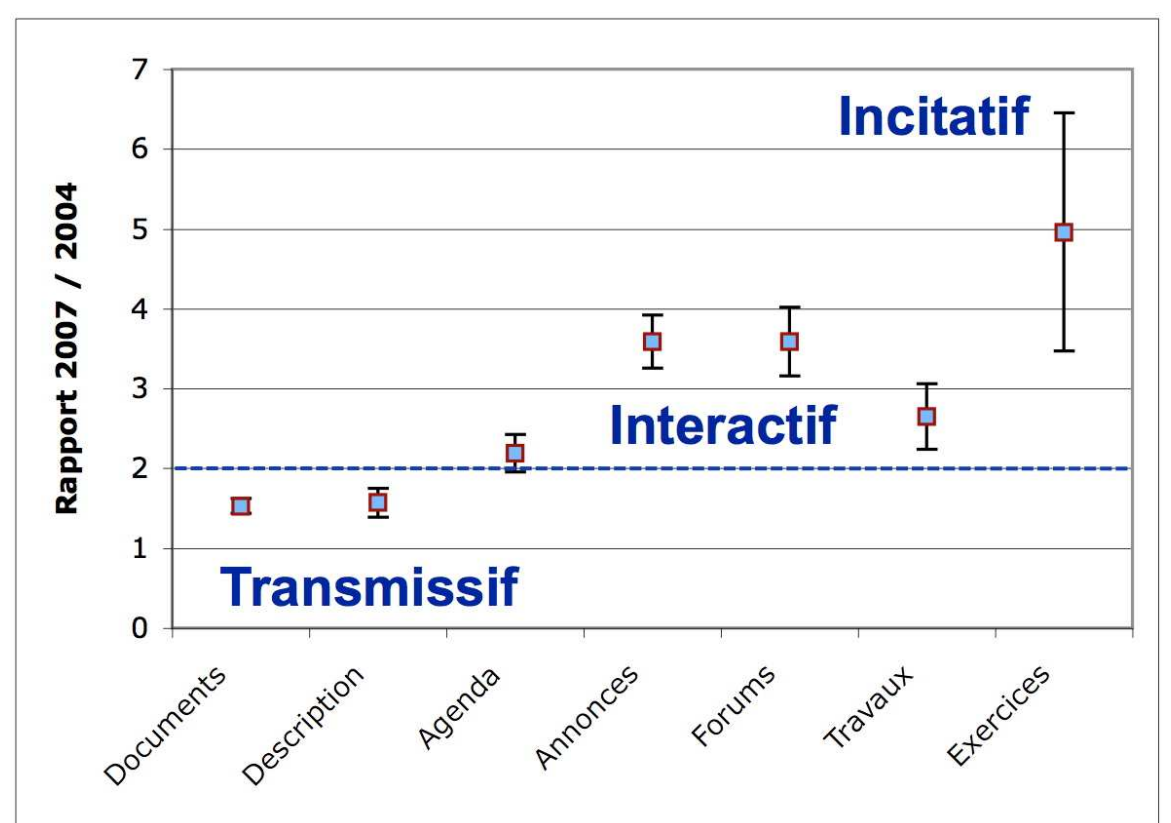

Figure 4. Rapports des usages de différents outils entre 2004 et 2007 (valeur attendue 2) et leurs erreurs statistiques

Dans une considération homogène, on pourrait s'attendre à ce que les indices globaux liés aux différents outils, tels que mesurés ici, aient également doublés en donnant un rapport entre 2007 et 2004 de 2 sur l'échelle verticale. Ces résultats nous paraissent intéressants dans le sens où ils présentent une stagnation si ce n'est un léger recul relatif des outils d'information (documents, description) et un renfort significatif, dans la limite des barres d'erreur, d'outils porteurs de davantage d'interaction (forums, par exemple) et d'incitation (exercices, par exemple).

Il s'agit ici d'un outil de mesure simple qui permet de monitorer l'évolution différentielle (les outils, les uns par rapport aux autres) et temporelle (au fil des années) des usages des différents outils. Il s'agit d'une information importante pour les responsables institutionnels à la recherche d'effets quantitatifs en rapport avec les investissements consentis.

Cependant, les effets observés ne peuvent être imputés aux seules présence et disponibilité des outils de la plateforme. Des facteurs comme un message institutionnel encourageant ces formes actives et interactives d'enseignement, comme la présence d'un institut de soutien aux enseignants (Service de pédagogie universitaire ou autre), comme la disponibilité d'un fonds de soutien aux innovations pédagogiques, comme des mesures de valorisation des efforts des enseignants innovateurs expliquent sans doute une partie de ces résultats. La méthodologie de recherche proposée ici est une sonde intéressante pour jauger les évolutions souhaitées et dont les résultats sont à exploiter et à préciser dans des recherches ultérieures. Il serait ainsi évidemment intéressant de reproduire une telle méthodologie dans d'autres lieux, dans d'autres contextes avec d'autres plateformes comme Moodle, Dokeos, Spiral ... Les évolutions dont nous parlons, liées à des conceptions relatives aux statuts des savoirs et aux rôles des formateurs, prennent un temps certain qu'il n'est pas aisé de quantifier : des recherches longitudinales portant sur plusieurs années sont à encourager. Ceci démontre la nécessité d'entreprendre des recherches amples, contextualisées (contextes des institutions, des programmes de formation et des référentiels de compétences associés, des méthodes mises en place...), ce qui nous semble hélas être éloigné des préceptes en vigueur dans les sciences fondamentales (définition et réduction des variables indépendantes et dépendantes, blocage des variables intermédiaires et contextuelles...). Comme nous le disons souvent «les étudiants ne sont pas des particules élémentaires »!

\subsection{Les effets des technologies sur l'apprentissage}

Comme nous l'avons dit dès l'introduction, de nombreuses recherches se sont penchées sur les effets des technologies sur l'apprentissage " assisté par ordinateur ». Vu le caractère interactif des variables liées 
aux technologies et aux pédagogies (Figure 1) ainsi que la difficulté de les isoler des contextes et d'isoler leurs composantes, les résultats sont souvent restés en demi-teinte. Par exemple, les recherches basées sur la comparaison des dispositifs avec ou sans technologies, ont montré que cette seule variable « indépendante » était relativement inopérante pour expliquer les variances des résultats. Le changement de surface apporté par les technologies ou au contraire les changements majeurs opérés dans le dispositif (objectifs, méthodes, rôles, ressources, évaluations) rendent la comparaison caduque ou alors ne permettent pas de se prononcer sur les effets attendus, trop de variables intermédiaires ou contextuelles noyant ces derniers.

Par rapport à cet état de fait, nous proposons ici deux approches concertées :

- Plutôt que de comparer des dispositifs (avec et sans technologies) finalement difficilement comparables (voir l'introduction), nous avons profité de la configuration hybride de notre institution (nos étudiants rencontrent sur une année académique ou tout au long de leurs études, des cours traditionnels transmissifs en amphi et aussi des dispositifs élaborés d'apprentissage sur plateforme) pour interroger directement les étudiants (et les enseignants) sur la valeur ajoutée (par la technologie) qu'ils percevaient quant à leurs apprentissages (étudiants) ou aux occasions d'apprentissages mis en place (enseignants)

- Plutôt que de travailler sur des variables, liées aux résultats obtenus aux interrogations et aux examens, trop sujettes à des variations peu ou difficilement contrôlables, nous nous sommes ainsi intéressés aux perceptions des étudiants quant aux apprentissages réalisés. Malgré son caractère subjectif, cette approche nous paraît être une mesure relativement directe (les items concernent des facteurs d'apprentissage et non les produits) et, comme les pédagogues de la motivation l'ont bien montré (Viau. 1994), ce sont pourtant ces perceptions de l'environnement qui in fine déterminent largement les apprentissages subséquents.

Il s'agit pour nous de tenter dépasser les résultats du no significant différence observé dans les méta-recherches citées en introduction.

\subsubsection{Approche globale}

Nous avons donc proposé, en 2006 et 2007, aux étudiants utilisateurs de la plateforme (ainsi qu'aux enseignants), 26 propositions portant sur des valeurs ajoutées des technologies quant aux apprentissages. Elles sont toutes rédigées en termes de «plus » ou « davantage » d'un dispositif en ligne par rapport à un enseignement «traditionnel » dans lequel l'enseignant n'utilise pas de plateforme : «Les tâches proposées aux étudiants sont plus intéressantes », "Les ressources que le professeur propose sont de natures plus variées », "Les étudiants sont davantage actifs quand le professeur utilise iCampus dans le cours », " Grâce à iCampus, j'ai davantage d'interactions avec le professeur », « Les évaluations sont plus souvent basées sur la production des étudiants » ... Cette approche vise donc à demander aux participants aux enquêtes de se prononcer directement sur les différences perçues plutôt que de mesurer ces éléments dans un dispositif « sans plateforme » et dans un autre dispositif « avec plateforme ». Ces 26 propositions sont réparties sur les 5 dimensions du modèle pragmatique d'apprentissage présenté plus haut (Figure 3) : les ressources (informations), les activités, les productions, les facteurs de motivation perçus et les interactions sollicitées dans le dispositif. C'est ainsi que dans les exemples d'items donnés ci-dessus, on retrouve successivement les dimensions : motivation, information, activités, interaction et productions.

Les résultats détaillés de cette recherche ont été présentés ailleurs (Docq et al., 2008). Pour une présentation concise des résultats, nous avons regroupé les modalités « tout à fait d'accord " et "plutôt d'accord » pour constituer un paramètre global d'accord. Ce sont les dimensions Informations (items 1, 3 et 4), Motivation (item 6) et Interaction (items 2, 24 et 25) qui émergent des résultats comme le précise le tableau ci-dessous (Tableau 3) dans lequel nous présentons en grisé les résultats des cinq items ayant reçu les plus hauts accords (pour les étudiants et les enseignants) quant aux valeurs ajoutées du dispositif en ligne que les participants à l'enquête devaient choisir.

\begin{tabular}{|l|l|l|}
\hline Items & $\begin{array}{l}\text { Pourcentage de sujets ayant } \\
\text { P : questionnaire « Professeur » } \\
\text { E : questionnaire «Etudiant » }\end{array}$ & $\begin{array}{l}\text { "tout à fait d'accord » } \\
\text { tout d'accord } » \text { ou }\end{array}$ \\
\hline
\end{tabular}




\section{Tableau 3. Items de valeurs ajoutées de la plateforme ayant reçu les accords les plus élevés}

Nous précisons encore une fois qu'il s'agit bien d'accords exprimés sur des valeurs ajoutées et non pas de comparaisons : la mesure directe que nous faisons est la perception de la différence entre le cours en ligne, objet de l'enquête choisi par le répondant, et un cours dans lequel l'enseignant n'utilise pas de plateforme.

Si nous analysons ces deux « top 5 » (en grisé), celui des enseignants et celui des étudiants, nous voyons se dégager quatre catégories de changements dont deux liées à des compétences liées au Lifelong Learning (compétence en recherche d'information, littéracie numérique et travail d'équipe, le «I » et le « $\mathrm{C} »$ des TIC) et deux liées à des dimensions motivationnelle et organisationnelle :

- Le développement de compétences liées à l'apprentissage tout au long de la vie : utiliser davantage les TIC (cité dans le top 5 des deux publics) et chercher des informations (cité par 64\% des étudiants).

- L'augmentation des interactions sociales : entre étudiants (cité dans le top 5 des deux publics) et avec les étudiants (du point de vue de 67\% des enseignants).

- Une augmentation de la motivation, chez $46 \%$ des étudiants.

- Une facilité logistique d'organisation des travaux de groupe, chez $54 \%$ des enseignants.

Ces recherches, antérieures de quelques années, sont à la fois complémentaires aux travaux récents de (Raby et al., 2011) en ce qui concerne, par exemple, les paramètres liés à la motivation et à l'acculturation technologique et relativement conformes en ce qui concerne les items liés à l'accès à l'information et aux interactions entre les enseignants et les étudiants et entre les étudiants. Ici, de nouveau, des différences significatives apparaissent entre les étudiants et les enseignants interrogés.

Les paramètres d'accord globaux (moyenne sur les 26 propositions) restent cependant un peu faibles, en tout cas en dessous de ce que pourraient attendre des évangélistes de la technologie éducative : $\mathbf{3 9 \%}$ pour les enseignants et $\mathbf{3 8 \%}$ pour les étudiants, un taux qui exprime le degré d'accord avec des impacts positifs des TIC sur l'apprentissage. On est encore loin de la révolution copernicienne annoncée, mais on progresse.

\subsubsection{Une analyse plus détaillée ... Un rapport entre technologies et pédagogies?}

Ces résultats globaux sont cependant entachés d'une variance importante liée aux dispositifs effectivement vécus par les étudiants ou alors effectivement mis en place par les enseignants (Lebrun et al., 2009). Il s'agit ici d'une perspective originale qui tente de nuancer les résultats des recherches en fonction des dispositifs effectivement vécus par les acteurs. C'est rarement le cas, vu la difficulté de catégoriser les dispositifs, et nous tentons d'apporter une solution pragmatique à cette nécessité. Nous avons abordé cette problématique de deux manières :

(a) Nous avons analysé le paramètre d'accord global (la moyenne sur les accords par rapport aux 26 propositions d'avantage de la plateforme technologique par rapport à des formes traditionnelles d'enseignement) en fonction du nombre d'outils de la plateforme connus et utilisés par les participants aux enquêtes. Cette méthode d'investigation reste large, les participants répondant éventuellement en intégrant probablement, par un effet de halo, une perception globale de plusieurs cours qu'ils ont expérimentés sur la plateforme, mais elle suffit, nous semble-t-il, dans cette approche exploratoire. 


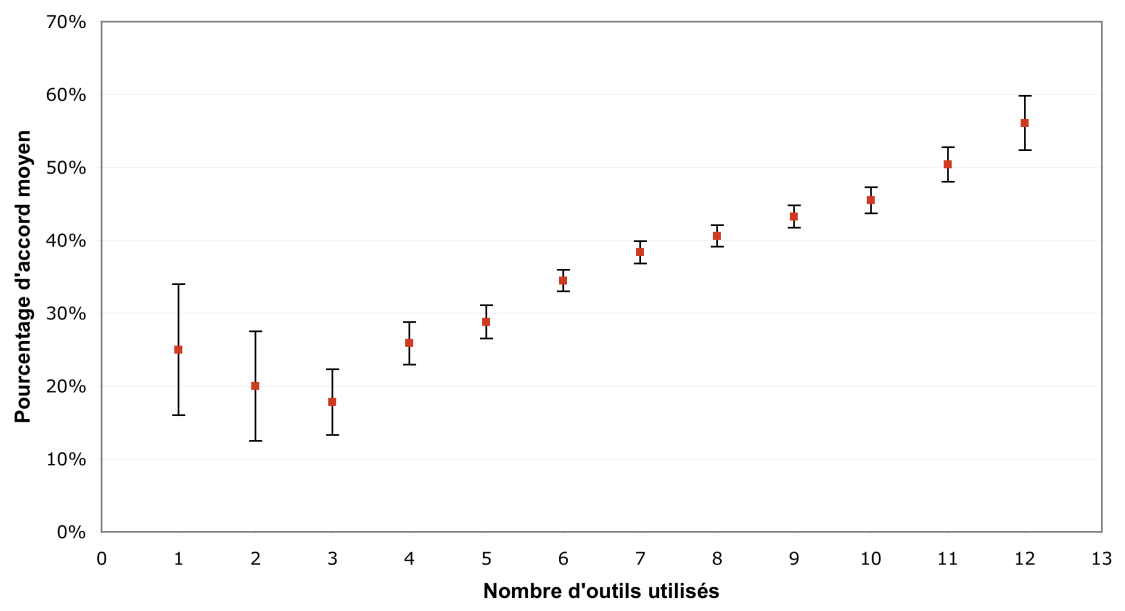

Figure 5. Perceptions des valeurs ajoutées pour l'apprentissage (étudiants)

Le graphique ci-dessus (Figure 5) présente (en ordonnée) ce paramètre d'accord global avec les valeurs ajoutées (les 26 propositions) de la plateforme en fonction du nombre d'outils connus et utilisés (en abscisse) par les étudiants, l'échelle allant de 1 (un seul outil utilisé) à 12 (tous les outils proposés ont été utilisés).

Une analyse plus fouillée montre que la partie gauche est surtout constituée d'outils de transmission, la partie médiane y ajoute principalement les outils d'interaction et la partie droite est constituée de l'ensemble des outils avec une part notable et supplémentaire des outils d'incitation. La recherche Hy-SUP, à laquelle nous contribuons, a produit récemment une typologie des dispositifs hybrides qui conforte cette gradation dans la richesse du dispositif : type 1, dispositif centré enseignement et acquisition de connaissances ; type 3 , dispositif centré enseignement mettant à disposition des outils d'interaction ; type 5, dispositif ouvert centré apprentissage ... (Burton et al.. 2011).

On remarquera que le paramètre d'accord global avoisine les $25 \%$ (accord sur les valeurs ajoutées) dans la partie gauche pour aller vers plus de 50\% dans la partie droite marquée par la richesse pédagogique des dispositifs vécus par les étudiants. L'enrichissement du dispositif est, selon nous, obtenu par une attention particulière à celui qui apprend (on dépasse l'enseignement transmissif que ce soit avec ou sans TIC) et aussi à une activation de facteurs d'apprentissage comme l'activité (l'incitation à l'activité) et l'interactivité (la mise à disposition de l'apprenant d'occasions de co-construction). Des auteurs fondamentaux (Dewey, Piaget, Vigotsky ...) ne nous contrediraient pas.

Il s'agit là d'un effet relativement « spectaculaire » par rapport aux effets ténus des recherches évoquées précédemment qui amalgament des dispositifs parfois très différents rencontrés par les étudiants. Ils démontrent l'importance de dépasser la variable indépendante " avec ou sans TIC » (le pôle « Technologies « de la Figure 1) pour tenir compte de ses interactions avec le dispositif pédagogique, élaboré ou non, construit « autour » ou « avec » l'outil (le pôle « Pédagogies » de la Figure 1).

(b) Mais, au-delà des outils utilisés, qu'en est-il des usages effectifs ? Même si «l'outil n'est que l'outil » et même si nous pouvons comprendre que les outils dont nous parlons induisent des usages (effet catalyseur), ce sont néanmoins ces derniers qui prévalent en termes d'apprentissage des étudiants : Our review provides convincing evidence that information technology can enhance learning when the pedagogy is sound, and where there is a good match of technology, techniques and objectives (Kadiyala et Crynes, 2000).

Nous avons abordé la question des usages au travers de 11 propositions (voir le tableau 2) qui décrivent des activités de natures transmissives ou réactives, incitatives ou encore interactives.

Le graphique ci-dessous (Figure 6) montre en abscisse ces usages (la numérotation est donnée au Tableau

2 ) et en ordonnée le paramètre global d'accord moyen sur les valeurs ajoutées pour l'apprentissage. 


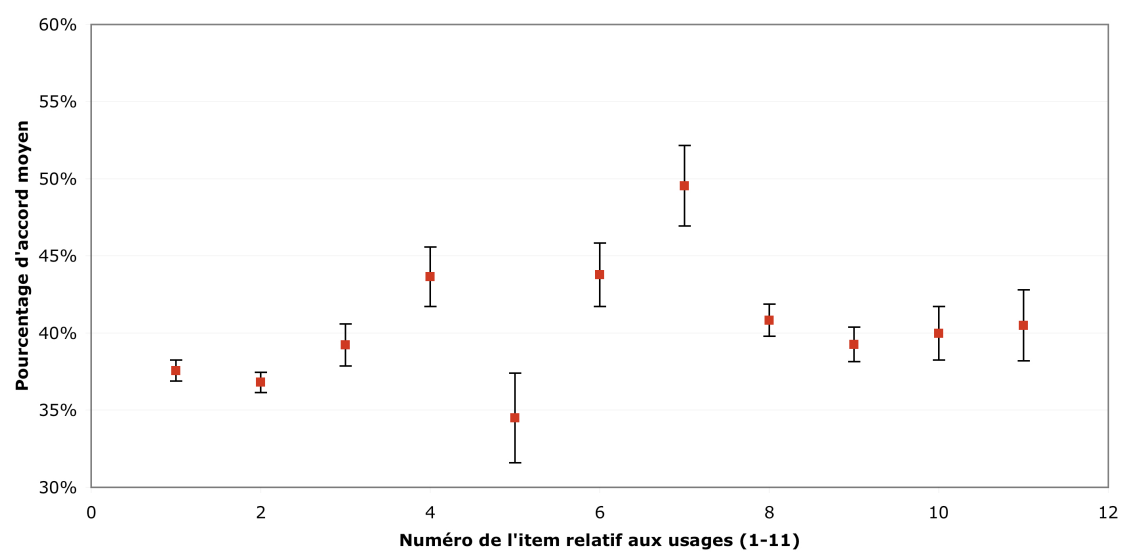

Figure 6. Usages de la plateforme (de 1 à 11 - Tableau 2) et perception globale des valeurs ajoutées pour l'apprentissage

Malgré la sensibilité légèrement moindre de cet indicateur par rapport à la densité d'outils utilisés dans le dispositif (voir Figure 5), on remarque cependant quelques items qui sortent sensiblement du lot (au-delà des barres d'erreur purement statistique) : l'item (4) " Communiquer avec le professeur », l'item (6) « Travailler en groupe avec d'autres étudiants » et l'item (7) « Maintenir la communication en dehors des activités en classe ».

Encore une fois, ce sont des ingrédients de l'un des deux «moteurs » du modèle pragmatique d'apprentissage qui sont mis en avant dans les éléments influençant la perception des valeurs ajoutées pour l'apprentissage : les modes d'interaction supportés par les outils. En particulier, l'item 7 démontre une des caractéristiques de l'hybridation : la possibilité de maintenir les interactions en dehors de l'activité de la classe. Un apprentissage sans distance promu par l'enseignement à distance ? Une composante majeure de l'apprentissage tout la vie durant? Les modifications de «l'espace-temps de l'apprentissage et de la formation » sont bien une des opportunités et en même temps un des effets des outils TIC dans l'univers de l'information et de la communication.

\subsection{Des effets sur l'apprentissage nuancés par la perception générale des TIC ?}

Au point précédent, nous avons observé un pourcentage d'accord moyen de 39\% (enseignants) et 38\% (étudiants) avec des propositions positives quant aux effets des TIC sur l'apprentissage. Nous venons de le voir, ces résultats moyens présentent des variations importantes relativement aux outils proposés et aux usages effectifs dans les dispositifs pédagogiques. Mais qu'en est-il de la dépendance de ces résultats (effets des TIC sur l'apprentissage) par rapport aux opinions générales des acteurs par rapport à ces technologies (voir le point 3.1) ? Comment la perception des impacts des TIC sur l'apprentissage est-elle influencée par de opinions plus générales comme « Cela coûte cher », « Les TIC prennent beaucoup de temps », « Je n'ai pas les compétences nécessaires » ? Cette question dans son ampleur fera l'objet d'une autre publication mais nous en donnons ici des résultats généraux résultants des enquêtes auprès des étudiants (en 2006, N=1179).

Le tableau ci-dessous (Tableau 4) présente les pourcentages d'accord moyens (sur les 26 propositions d'effets positifs sur l'apprentissage de la plateforme iCampus) exprimés par les étudiants répartis en quatre catégories : ceux en (1) accord ou en (2) désaccord sur les (1) appuis et les (2) obstacles.

\begin{tabular}{|l||l||l|}
\hline $\begin{array}{l}\text { Pourcentage } \\
\text { d'accord avec les } \\
\text { valeurs ajoutées } \\
\text { pour l'apprentissage }\end{array}$ & $\begin{array}{l}\text { Etudiants } \\
\text { désaccord }\end{array}$ & Etudiants en accord \\
\hline \hline Items-Obstacles & $38,9+/-0,3 \%$ & $35,8+/-0,4 \%$ \\
\hline
\end{tabular}




\begin{tabular}{|l|l|l|}
\hline Items-Appuis & $31,2+/-0,4 \%$ & $42,9+/-0,3 \%$ \\
\hline
\end{tabular}

\section{Tableau 4. Pourcentages d'accord avec les propositions d'effets positifs des TIC sur l'apprentissage pour quatre catégories d'opinions générales sur les TIC}

Nous l'avions vu précédemment (Tableau 1) : environ un tiers de nos étudiants étaient réservés quant au fait que les technologies puissent présenter un appui, un avantage pour la formation ... ils montrent également le plus faible pourcentage d'accord avec les propositions de valeurs ajoutées des technologies pour l'apprentissage (31,2\%). Les étudiants en accord, avec les appuis généraux présentent, quant à eux, les accords les plus élevés avec les valeurs ajoutées des TIC pour l'apprentissage (42,9\%). Rappelons que si les obstacles déclarés par rapport à l'utilisation des TIC se sont effondrés entre 2001 et 2006-2007, les perceptions des appuis que les technologies présentent n'ont pas connu une réelle progression (point 3.1). Au-delà des formations techniques auxquelles on pourrait penser pour sensibiliser les étudiants à l'utilisation des TIC pour leurs apprentissages (l'aisance par rapport aux technologies et les compétences nécessaires constituant de moins en moins un problème), nous pensons à de véritables formations conduisant à une appropriation des usages (des routines) ainsi qu'à une acculturation numérique progressive dans les études. A l'heure de l'apprendre à apprendre, à quand des formations, non pas seulement sur les outils mais, sur l'apprentissage avec les TIC, à quand des formations à l'apprentissage « tout court »? Selon nous, qu'il s'agisse des méthodes pédagogiques mises en place pour développer des compétences (demander un rapport scientifique aux étudiants pour qu'ils expérimentent la recherche d'informations, les faire travailler en groupe pour qu'ils développent la compétence au travail d'équipe) ou qu'il s'agisse de l'utilisation des TIC, on pense trop souvent qu'il suffit de plonger l'étudiant dans le dispositif pour qu'il apprenne. Le Learning by doing a ses limites. S'il s'agit de développer des compétences, une formation à la méthode ou à l'usage s'impose et une validation de la compétence développée est nécessaire.

\subsection{Des outils simples?}

Dans le contexte décrit plus haut et de notre hypothèse générale, nous avons présenté le fait que le développement de l'outil Claroline était inspiré par deux considérations : une impulsion institutionnelle pour promouvoir des apprentissages de qualité et le souhait d'offrir des outils simples pour que les enseignants puissent se consacrer, au-delà des difficultés techniques, à ce qui finalement importe le plus : l'apprentissage des étudiants. Est-ce le cas?

Ces considérations ne sont qu'une approche de variables qu'il nous paraîtrait aussi utile de considérer, simultanément à celles présentées ici, dans la recherche des effets des TIC : utilité et utilisabilité perçues, efficacité et efficience ... (Davis, 1989).

Dans l'enquête dont nous avons déjà parlé (2007) et dont la méthodologie a été décrite dans un article antérieur (Docq et al., 2008), 153 enseignants volontaires se sont exprimés à propos des mobiles qui les poussent à utiliser la plateforme de 1'UCL. Précisément la question était : " Quelles sont les (quatre) raisons les plus importantes qui vous ont motivé à adopter iCampus (le nom donné à la plateforme Claroline dans notre institution) dans votre enseignement? »

Parmi les 11 raisons proposées, on trouve des éléments de nature personnelle (goût des technologies), pédagogique (rendre les pédagogies plus actives), institutionnelle (souhait pressenti des autorités), en résumé, des motivations tant internes qu'externes.

Les quatre raisons les plus souvent citées sont (dans l'ordre d'importance, les fréquences s'échelonnant de 80 à $60 \%$ ):

- Pour simplifier la mise en place de mon enseignement

- Suite à la facilité d'utilisation d'iCampus

- Dans l'intention d'améliorer l'apprentissage des étudiants

- Pour aller vers des pédagogies plus actives. 
Notre hypothèse du rôle inducteur de technologies simples et intuitives pour l'amélioration de la pédagogie s'en trouve confortée, mais demandera encore de nombreux travaux ultérieurs. Associés aux résultats relatifs aux modifications des usages au cours du temps (présentés aux points 3.2 et 3.3), ces derniers confortent notre hypothèse générale (présentée à la fin du point 2.1) sur la migration (catalysée ou accélérée par les technologies) des enseignants vers des dispositifs pédagogiques plus riches, c'està-dire composés d'outils variés et dynamisés par des usages plus interactifs et plus proactifs des différents acteurs (point 3.4.2).

\section{Conclusions}

Nombreuses sont les discours et les publications qui prétendent, souvent de manière triomphale, à un impact positif des technologies sur la qualité des enseignements et des apprentissages. Ils sont généralement bâtis sur la supposition que les utilisations efficaces des technologies, accompagnées dès lors de méthodes pédagogiques plus actives ou interactives, devraient avoir un tel effet.

La condition nécessaire technologique est non suffisante. Plus rares (inexistantes à notre avis) sont les productions scientifiques qui ont tenté d'étayer cette supposition et d'en analyser les relations entre les constituants technologies et pédagogiques (Figure 1). Sans cette analyse fine, les résultats généraux les plus fréquents conduisent bien souvent au phénomène du No Significant Difference, pénalisant pour la promotion institutionnelle et sociétale de ces activités technopédagogiques ou alors à des résultats positifs difficilement reproductibles et parfois accidentels. Nous avons voulu contribuer à une approche pragmatique et empirique à la fois au niveau des données recueillies, des domaines investigués simultanément (par exemple, à la fois, les outils, les usages et les effets mesurés chez les étudiants et les enseignants) et longitudinalement et, des méthodologies de recherche mises en place.

(a) Une étude des perceptions générales des TIC (le coût, le temps, les compétences requises, la formation nécessaire ...) nous semble préalable et indispensable pour cadrer les effets éventuels des TIC sur les facteurs d'apprentissage. Ils conduisent (point 3.1) au constat que l'acculturation numérique progresse en constatant les résultats des recherches plus récentes par rapport aux recherches antérieures effectuées dès 2000-2001 (Reding et al., 2001). Néanmoins, on peut en conclure que « apprendre avec le numérique » est une préoccupation qui devrait percoler des recherches actuelles pour atteindre les pratiques et la formation de étudiants et des enseignants. À quand des formations pour " apprendre avec les technologies »? À quand des formations pour apprendre à l'ère du numérique ?

(b) Notre recueil de données sur les usages des outils de la plateforme Claroline à l'UCL (point 3.2) a montré, au cours des années, un (lent) mouvement statistiquement valide entre des usages plus traditionnels basés sur la transmission (dépôt de documents, d'informations relatives aux contenus et à l'organisation...) vers des usages à caractère plus interactif, on apprend avec les autres, ou plus incitatif, proactif, on apprend « en soi » (point 3.3). Ces études longitudinales devraient se déployer ailleurs, en d'autres contextes et sur d'autres plateformes afin de consolider nos conclusions. C'est un des buts de la recherche Hy-SUP dont nous avons parlé.

(c) Mesurer l'impact pédagogique de tels outils est difficile. Nous nous sommes penchés ici principalement sur la perception des étudiants et des enseignants quant aux apprentissages (point 3.4), en nous basant sur les cinq dimensions du modèle pragmatique d'apprentissage que nous avons proposé dès 1995 et que nous avons présenté plus haut. Comment pourrait-on mesurer des impacts sur les apprentissages sans disposer d'un modèle d'apprentissage, sans disposer d'une jauge pour le mesurer dans ces diverses dimensions ? Même si ces perceptions de valeurs ajoutées d'une plateforme sur l'apprentissage restent modestes (un pourcentage moyen d'accord avec les propositions de valeurs ajoutées pédagogiques d'une plateforme d'apprentissage en ligne d'environ $38 \%$ ), nous avons montré qu'elles dépendaient de la richesse (la variété et la complémentarité des outils et des usages) du dispositif pédagogique mis en place par l'enseignant et des composantes motivationnelles et interactives. Ici aussi, il serait intéressant de répéter ces mesures au cours du temps, en d'autres lieux. Cet impact pédagogique doit également être analysé en fonction de l'appropriation, par les différents acteurs, de la culture numérique (point 3.5).

Cette synthèse de travaux réalisés dans le cadre de l'Institut de Pédagogie universitaire et des Multimédias 
de l'Université catholique de Louvain à Louvain-la-Neuve apporte des éléments qui corroborent l'hypothèse générale d'impacts des TIC sur le développement professionnel des enseignants et, au-delà, sur la perception, par les étudiants, de la qualité des apprentissages effectués. Des instruments de mesure concrets sont aussi proposés afin de mesurer ces impacts. Conscients des limites inhérentes à ce travail prospectif, liées au grand nombre de variables intermédiaires et contextuelles soulignées dans cet article, nous apportons ainsi des éléments concrets à la question du rôle catalyseur des TIC dans le cadre de l'innovation dans l'enseignement et de la qualité des apprentissages : une plateforme d'eLearning, simple, intuitive et ouverte à des modalités variées d'enseignement constitue-t-elle un outil de formation pédagogique et de développement professionnel pour les enseignants ?

Les recherches présentées ici et le cadre ample multi-factoriel que nous suggérons pour mieux comprendre les effets des TIC sur l'apprentissage des étudiants et le développement professionnel des enseignants, trouveront encore des développements dans le cadre des recherches, menées au niveau européen, par le collectif Hy-SUP à propos des dispositifs hybrides (typologie et effets). Une première partie de cette recherche a conduit à une typologie de ces dispositifs (Burton et al.. 2011) qui confirment nos données antérieures (Lebrun et al., 2009). La deuxième partie consacrée aux déterminants et aux effets de ces dispositifs est en cours. Les résultats attendus permettront sans nul doute de consolider les dimensions proposées dans ce document et d'étendre la portée compréhensive des démarches entreprises ici.

\section{BIBLIOGRAPHIE et WEBOGRAPHIE}

BARRETTE, C. (2005). Vers une métasynthèse des impacts des TIC sur l'apprentissage et l'enseignement dans les établissements du réseau collégial québécois. Mise en perspective. Clic, 57, p. 18-24. Disponible sur Internet : http://bit.ly/50gcAj (Consulté le 27 septembre 2011).

BIGGS, J. (1996). Enhancing teaching through constructive alignment. Higher Education Vol. 32, p. 347-364.

BURTON, R., BORRUAT, S., CHARLIER, B., COLTICE, N., DESCHRYVER, N., DOCQ, F., ENEAU, J., GUEUDET, G., LAMEUL, G., LEBRUN, M., LIETARD, A., NAGELS, M., PERAYA, D., ROSSIER, A;, RENNEBOOG, E., VILLIOT-

LECLERCQ, E. (2011). Vers une typologie des dispositifs hybrides de formation en enseignement supérieur. Distances et savoirs, Vol. 1(9), p. 69-96. Disponible sur Internet : http://www.cairn.info/revue-distances-et-savoirs-2011-1-page-69.htm (Consulté le 27 septembre 2011).

BROWN, G. et ATKINS, M. (1988). Effective teaching in higher education. London : Routledge.

CARON, P.-A., \& VARGA, R. (2009). Artefacts malléables et perméables (AMP) pour mener des activités pédagogiques: L'usager acteur et créateur de son dispositif numérique. Distances et Savoirs, Vol. 7(2), p. 155-177.

CHARLIER, B., DESCHRYVER, N., et PERAYA, D. (2006). Apprendre en présence et à distance : Une définition des dispositifs hybrides. Distances et savoirs, Vol. 4(4), p. 469-496.

CLARK, R.E. (1983). Reconsidering research on learning from media. Review of Educational Research. Vol. 17(2), p. 92-101

BLOOM, B.S. (1956). Taxonomy of educational objectives : The classification of educational goals : Handbook I, Cognitive Domain. New York : Longmans.

DAVIS, F. D. (1989). Perceived usefulness, perceived ease of use, and user acceptance of information technology. MIS Quarterly, Vol. 13(3), p. 319-339.

DE PRAETERE, T. (2003). Claroline à l'UCL, histoire d'un développement. Disponible sur Internet : http://www.dokeos.com /fr/node/579 (Consulté le 27 septembre 2011).

DOCQ, F., LEBRUN, M. et SMIDTS, D. (2008). Analyse des effets de l'enseignement hybride à l'université : détermination de critères et d'indicateurs de valeurs ajoutées. Revue internationale des technologies en pédagogie universitaire, Vol. 7(3), p. 48-59. Disponible sur Internet : http://www.ritpu.org/IMG/pdf/RITPU_v07_n03_47.pdf (Consulté le 27 septembre 2011).

Docq, F., Lebrun, M. et Smidts, D. (2010). À la recherche des effets d'une plateforme d'enseignement/ apprentissage en ligne sur les pratiques pédagogiques d'une université : premières approches. Revue internationale des technologies en pédagogie universitaire, Vol. 5(1), p. 45-57. Disponible sur Internet : http://www.ritpu.org/IMG/pdf/RITPU v05n01 45.pdf (Consulté le 27 septembre 2011).

HENRI, F. et LUNDGREN-CAYROL, K. (2001). Apprentissage collaboratif à distance. Pour comprendre et concevoir les environnements d'apprentissage virtuels. Sainte-Foy, Québec: Presses Universitaires du Québec.

KADIYALA, M., et CRYNES, B.L. (2000). A review of literature on effectiveness of use of Information Technology in 
Education. Journal of engineering education, Vol. 89(2), p. 177-190.

KULIK, J., KULIK, C., et COHEN, P. (1980). Effectiveness of computer-based college teaching : A meta-analysis of findings. Review of Educational Research, Vol. 50, p. 525-544.

LAMEUL, G. (2008). Les effets de l'usage des technologies d'information et de communication en formation d'enseignants sur la construction des postures professionnelles. Savoirs, Vol. 17, p. 73-94.

LEBRUN, M. et VIGANÒ, R. (1995a). De l'« Educational Technology » à la technologie pour l'éducation. Cahiers de la recherche en éducation, Sherbrooke (Canada), Vol. 2(3), p. 267-294.

LEBRUN, M. et VIGANÒ, R. (1995b). Des multimédias pour l'éducation : de l'interactivité fonctionnelle à l'interactivité relationnelle. Cahiers de la recherche en éducation, Sherbrooke (Canada), Vol. 2(5), p. 457-483.

LEBRUN, M. (2004). Enseigner et apprendre en ligne. Claroline et le site iCampus de l'UCL : fondements, outils, dispositifs, in S. Alava, Formation ouverte et à distance : actualités de la recherche. Le dossier des sciences de l'éducation, Vol.12.

LEBRUN, M. (2005a). eLearning pour enseigner et apprendre : Allier pédagogie et technologie. Louvain-la-Neuve : AcademiaBruylant.

LEBRUN, M. (2005b). Théories et méthodes pédagogiques pour enseigner et apprendre : Quelle place pour les TIC dans l'éducation? 2ème édition revue. Bruxelles : De Boeck.

LEBRUN, M. (2007). Quality towards an expected harmony: Pedagogy and technology speaking together about innovation. AACE Journal, Vol. 15(2), p. 115-130. Chesapeake, VA: AACE. Disponible sur Internet : http://www.editlib.org/p/21024 (Consulté le 27 septembre 2011).

LEBRUN, M. (2008). SOTL as a learning process. Document non publié de l'Institut de Pédagogie universitaire et des multimédias (IPM) de l'Université catholique de Louvain (UCL). Disponible sur Internet : http://bit.ly/SOTL-Lebrun (Consulté le 27 septembre 2011).

LEBRUN, M. (2011). Dispositif hybride et flipped classrooms. Blog de M@rcel. Disponible sur Internet : http://lebrunremy.be /WordPress/?p=583 (Consulté le 15 octobre 2011).

LEBRUN, M., DOCQ, F. et SMIDTS, D. (2009). Claroline, an Internet Teaching and Learning Platform to Foster Teachers' Professional Development and Improve Teaching Quality : First Approaches. AACE Journal, Vol. 17(4), p. 347-362. Chesapeake, VA: AACE. Disponible sur Internet : http://www.editlib.org/p/29355 (Consulté le 27 septembre 2011)

LESNE, M. (1977). Travail pédagogique et formation d'adultes. Paris : PUF.

MEUNIER, J.-P. et PERAYA, D. (2004). Introduction aux théories de la communication. Bruxelles: De Boeck.

MORGAN, G. (2003). Faculty use of course management systems. Research Study from the EDUCAUSE Center for Applied Research. Disponible sur Internet : http://connect.educause.edu/Library/ECAR/FacultyUseofCourseManagem/39089 (Consulté le 27 septembre 2011).

RABARDEL P. (1995). Les hommes et les technologies, approche cognitive des instruments contemporains. Paris : Armand Colin.

RABY, C., KARSENTI, T., MEUNIER, H. et VILLENEUVE, S. (2011). Usage des TIC en pédagogie universitaire : point de vue des étudiants. Revue internationale des technologies en pédagogie universitaire, Vol. 8(3), p. 6-19. Disponible sur Internet : http://www.ritpu.org/IMG/IMG/pdf/RITPU_v08_n03_6.pdf (Consulté le 27 septembre 2011).

REDING, R., DENEF, J.-F., PARMENTIER, P. et LEBRUN, M. (2001). Accès, compétences et opinions des étudiants en médecine vis à vis des technologies de l'information et de la communication. Pédagogie Médicale, Vol. 2(4).

RUSSELL, T.L. (2009). The no significant difference phenomenon. Chapel Hill, NC : Office of Instructional Telecommunications, North Carolina State University. Disponible sur Internet : http://www.nosignificantdifference.org/ (Consulté le 27 septembre 2011).

TARDIF, J. (1996). Une condition incontournable aux promesses des NTIC en apprentissage : une pédagogie rigoureuse. Actes de la Conférence d'ouverture au colloque de l'AQUOPS (Association québécoise des utilisateurs de l'ordinateur au primaire et au secondaire).

VIAU, R. (1994). La motivation en contexte scolaire. Bruxelles : De Boeck.

VOOGT, J. et KNEZEK, G. (Eds.) (2008). International Handbook of Information Technology in Primary and Secondary Education. New York: Springer.

1 Le projet HY-SUP 2009-2012 (DG. Education et Culture. Programme pour l'éducation et la formation tout au long de la vie) est coordonné par l’Université Claude Bernard Lyon 1 (E. Bettler et N. Coltice). Ce 
projet associe des chercheur-e-s et enseignant-es des universités de Fribourg (B. Charlier et A. Rossier), de Genève (N. Deschryver, C. Peltier, D. Peraya, A. Ronchi et E. Villiot-Leclercq), de Louvain-La-Neuve (F. Docq, M. Lebrun, C. Letor et A. Liétart), de Lyon 1 (C. Batier et C. Douzet), de Luxembourg (R. Burton, G. Mancuso et E.Renneboog) et de Rennes 2 (B. Albero, J. Eneau, G. Gueudet, G. Lameul, M. Nagels).

$\underline{2}$ L'auteur remercie ses collègues de l'IPM, Françoise Docq et Denis Smidts pour leur soutien et leurs apports dans les recherches communes présentées ici et dont cet article constitue une synthèse.

Référence de l'article :

Marcel LEBRUN (UCL, Louvain-la-Neuve, Belgique), Impacts des TIC sur la qualité des apprentissages des étudiants et le développement professionnel des enseignants : vers une approche systémique, Revue STICEF, Volume 18, 2011, ISSN : 1764-7223, mis en ligne le 16/11/2011, http://sticef.org

(C) Revue Sciences et Technologies de l'Information et de la Communication pour l'Éducation et la Formation, 2011 